\title{
Using Branch-Grafted R-trees for Spatial Data Mining
}

\author{
Priyanka Dubey ${ }^{1}$, Zhengxin Chen ${ }^{1}$, and Yong Shi ${ }^{1,2}$ \\ ${ }^{1}$ College of Information Science and Technology \\ University of Nebraska at Omaha \\ Omaha, NE 68182, U. S. A. \\ \{zchen,yshi\} @mail.unomaha.edu \\ ${ }^{2}$ Graduate School of Chinese Academy of Sciences, Beijing 100039, China
}

\begin{abstract}
Spatial data mining is a process of extraction of implicit information, such as weather patterns around latitudes, spatial features in a region, etc., with a goal of knowledge discovery. The work reported here is based on our earlier work on branch-grafted $\mathrm{R}$ trees. We have taken a bottom-up approach in our research: from efficient spatial data structure (i.e., branch-grafted $\mathrm{R}$ tree implementation), to efficient data access methods, and finally, to effective spatial data mining. Since previous experiments have shown that there are significant advantages of using branch-grafted implementation, this bottom-up approach exploits the performance advantages of the branch-grafted R-trees.
\end{abstract}

\section{Introduction}

Spatial data mining [4] refers to the extraction of implicit knowledge, spatial relationships, or other patterns, not explicitly stored in databases involving spatial data. Spatial data differs from data stored in relational databases. It carries topological information usually organized by multidimensional indexing structures, accessed by spatial data access methods. The focus of this paper is on using branchgrafted R-tree for data mining. Nevertheless, in order to make this paper selfcontained, we also provide relevant background related to $\mathrm{R}$ trees and their branchgrafted implementation. Therefore, space limitation will only allow us to provide a general description of our approach, leaving much of the details to future papers.

We start our discussion with a brief review of necessary background. R-trees [2] are specialized forms of $\mathrm{B}+$ trees adapted for the efficient representation, access and management of spatial data. An R-tree is a height-balanced tree that contains all leaves at the same level. The root node has at least two children. The non-leaf nodes contain $\lceil\mathrm{m} / 2\rceil$ entries, where $\mathrm{m}$ is the maximum number of entries in a node. The spatial data is represented as rectangles called minimum bounding rectangle. A nonleaf node contains information of the bounding rectangle, in the form of the coordinates, of its child node. The leaf node of an R-tree stores the record of spatial data stored, in the form of the coordinates of a rectangle, in relational database. Although the original R-tree structure has many merits, it also leaves rooms for improvement. Variations of R-trees exist (such as $\mathrm{R}^{*}$ tree). As an improvement proposed from our research group, the branch grafted algorithm uses the R-tree with 
better reorganization of records. The algorithm uses the grafting of a leaf or a branch node in the tree to reduce the number of nodes in the R-tree. The more accurate data structure improves the performance of query. The split in the original R-tree does not consider rest of the nodes in the tree while the branch-grafted tree scans the other nodes in order to find an opening. This opening can accommodate a rectangle. This could avoid the need of a split and reduces the number of node in the tree. The improvement in search time is a result of lesser number of nodes in the tree.

The branch-grafting algorithm improves the accuracy and speed of the node overflow operations. In case of a node overflow, the Branch Grafting algorithm first looks for records in the parent node that overlap the bounding directory of the full node. Individual records in the full node are then evaluated for placement under the overlapping parent nodes. Records are moved under a parent node if the resulting area of coverage for all nodes involved is smaller. If no records are moved to make room for the initially inserted record, then a split is performed using the original R-tree split algorithm.

Branch-grafted R-trees have been used to deal with location-based regionalqueries and point-queries. Branch-grafted R-trees have an advantage when a huge amount of spatial data needs to be stored. Grafting reduces the height of the tree, which helps the data access from the tree. A regional query finds all data objects that intersect a given spatial rectangle formed around a spatial object that overlap or fall within the query window. A point-query is a special case of the region query in which an aligned rectangle is a single point. The data access is faster as the search algorithm traverses fewer search paths due to smaller number of nodes reduced by grafting.

Once the spatial data is organized using an efficient data structure such as branch grafted R-tree, data access mechanisms should be developed for further processing. Basic spatial data access methods developed in earlier work of our institution $[1,3,6]$ include window search algorithm (also called overlap algorithm, which finds all the objects that overlap a specified search window and can be used for location-based queries) and its variation window search algorithm (also referred to as contains algorithm), search by distance algorithm and its extension nearest neighbor algorithm, etc.

\section{Extending Spatial Queries for Spatial Data Mining}

The representation and analysis of the information obtained as result of spatial queries, is important for the better understanding, usage, and meaningful insight into the spatial data. Recent studies of mining spatial rules have led to a set of interesting techniques to represent the patterns and features in a spatial data set. Strong spatial rules indicate the patterns and implication relationships in the large spatial data set. We can distinguish several kinds of spatial data mining rules:

- Spatial Association Rules: Spatial association is a rule that describes the implication of one or a set of features by another set of features in spatial database. 
- Spatial Classification Rules: The process of spatial classification is to find a rule to partition a set of classified objects into a number of classes using spatial relations of the classified objects to other objects in the database.

- Spatial Aggregation Rules: Aggregate values for areas close to spatial objects plays a very important role in the analysis of many business objects like stores, gas stations, etc.

- Spatial Discriminant Rules: A spatial discriminant rule is a general description of the contrasting or discriminating features of a class of spatial-related data from other classes.

\section{Spatial Data Mining Program Construction and Experiments}

The spatial data mining program consists of three modules.

- Module 1 builds a data structure using input data files and stores the data in the branch grafted R-Tree.

- Module 2 is designed to retrieve relevant data stored in the branch grafted R-Tree with the help of queries and stores the relevant output in a text file.

- Module 3 describes the mining of the spatial rules viz. spatial association rule, aggregation rule, and discriminant rules from the pool of matching coordinates.

The main spatial operators used for the query design in the research can be mainly divided in two categories; topological and directional. The spatial operators such as 'Containment', 'intersection', 'adjacency', 'inside', and 'within' fall under the category of topological operators, while 'Northwest', 'distance_compared_to', 'near', 'close_to' are directional operators.

An example of spatial query that uses 'adjacent_to' function is shown below:

SELECT CITY_NAME, CITY_LOCATION

FROM CITY, HYDRO

WHERE CITY_LOCATION ADJACENT_TO (WATER_BODY)

AND CLOSE_TO(US BUNDARY,30) AND

'ATLANTIC_OCEAN'

WATER_BODY =

The spatial data for the work has been down loaded from TIGER/ LINE. The TIGER database contains spatial information about hydrology, transportation, and other objects like national and state parks, churches, universities etc. The data used in this work deals water bodies in the North America, cities of North America, and US highways. More information about cities by counties was obtained from LANDVIEW3 site. This data contains the spatial and detailed non-spatial information as, census, industries, income level, etc. The raw data from different counties and states was rearranged and merged to make a master data file. The data format is as follows:

minimum x coordinate, minimum y coordinate, positive delta $\mathrm{x}$, positive delta $\mathrm{y}$, name

Several data sets have been used for experiments. For example, the data set hydro_tiger95 contains hydrographic spatial data, with number of records 360,330 . 
Below are some spatial rules discovered by the implemented program:

\section{Spatial Association Rules}

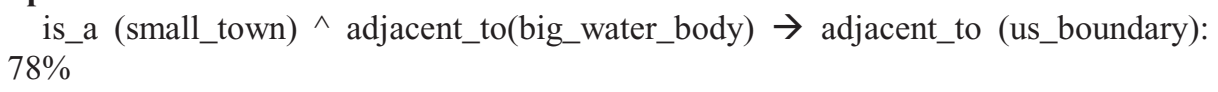

\section{Spatial Aggregation Rules}

Total number of small cities close_to(big_water_bodies) in North America: 134 Spatial Discriminant Rules

Large number of industries are located within(big_cities) while small number of cities located within(small_cities)

\section{Conclusion}

As indicated in our discussion, we have taken a bottom-up approach in our research: from efficient spatial data structure (i.e., branch-grafted $\mathrm{R}$ tree implementation) [1,6], to efficient data access methods [3], and finally, to effective spatial data mining [5]. Since previous experiments have shown that there are significant advantages of using branchgrafted implementation, this bottom-up approach exploits the performance advantages of the branch-grafted R-trees. However, due to space limitation, detailed description of algorithms, experiments and their analysis are omitted here. As for the future work, more experiments on larger data sets and more detailed analysis are still needed.

\section{References}

1. B. Asato, Branch-Grafting Heuristic for R-tree Implementation, Working Paper, Department of Computer Science, University of Nebraska at Omaha, 1994.

2. A. Guttman, R-trees: A Dynamic Index Structure for Spatial Searching, Proc. ACM IGMOD, 47-57, 1984

3. M. Khanijo, Z. Chen and Q. Zhu, Spatial data access methods using branch-grafted Rtrees, Advances in Database and Knowledge-Based Systems: Data Mining and Data Warehousing (G. E. Lasker and Z. Chen eds.), 31-36, 2000.

4. K. Koperski, J. Han, and J. Adhikary, Mining Knowledge in Geographical Data, IEEE Computer, 1998.

5. P. Dubey, Spatial Data Mining Using Branch-Grafted R-tree, MS thesis, University of Nebraska at Omaha, Omaha, 2003.

6. T. Schreck and Z. Chen, Branch grafting method for R-tree implementation, Journal of Systems and Software, 53(1), 83-93, 2000. 